\title{
AdAPTIVE LEARNING MANAgEMENT System USING SEMANTIC WEB TECHNOLOGIES
}

\author{
M.Farida Begam ${ }^{1}$ and Gopinath Ganapathy ${ }^{2}$ \\ ${ }^{1}$ Department of Information Technology, Manipal University Dubai, U.A.E, \\ farida@manipaldubai.com \\ ${ }^{2}$ School of Computer Science and Engineering, Bharathidasan University, India \\ gopinath.g@csbdu.in, drgg@bdu.ac.in
}

\begin{abstract}
Ontologies and semantic web services are the basics of next generation semantic web. This upcoming technologies are useful in many fields such as bioinformatics, business collaboration, Data integration and etc. E-learning is also the field in which semantic web technologies can be used to provide dynamism in learning methodologies. E-learning includes set of tasks which may be instructional design, content development, authoring, delivery, assessment, feedback and etc. that can be sequenced and composed as workflow. Web based Learning Management Systems should concentrate on how to satisfy the e-learners requirements. In this paper we have suggested the theoretical framework ALMS-Adaptive Learning management System which focuses on three aspects 1) Extracting the knowledge from the use's interaction, behaviour and actions and translate them into semantics which are represented as Ontologies 2) Find the Learner style from the knowledge base and 3)deriving and composing the workflow depending upon the learner style. The intelligent agents are used in each module of the framework to perform reasoning and finally the personalized workflow for the e-learner has been recommended.
\end{abstract}

\section{KEYWORDS}

Learning Style, Learning Objects, workflow, web services, Semantics, Ontology, LMS, ALMS, and OWL-S

\section{INTRODUCTION AND OBJECTIVE}

Many Universities across the world began their on line learning management system. Traditional teacher-learner centric classes are converted to repeated learner centric courses where reusability is enforced and time, space constraints are broken. Now many learners both students and academicians believe in continuous learning-teaching processes which rely on world wide web. Each learner has his/her own traits pace, prior knowledge level in the course, and requirements. Semantic web based learning management system which is adaptive in terms of finding the learners style and provides learning objects or services according to the style of the learner dynamically is the requirement now a days. This kind of LMS that allows learners to determine their learning agenda and control their own Learning is a current necessity. Learners understand and assimilate the concepts learned and apply them in practical solutions. Latest and Future learning systems have to consider this customized learning paths to their design and implementation. Dynamic Composition of e-Learning contents and e-learning services and Semantic Querying for Learning materials and constructing learners preferred courses are the DOI: $10.5121 /$ ijsc.2013.4101 
features which to be implemented in the any adaptive e-Learning management system. This process involves building knowledge base and detecting learner's style and composition of workflow of e-learning services. The main objective of the e-learning framework which is proposed in this paper is to develop a completely new, semantically enhanced, automatically composed adaptive e-learning system for dynamic learners.

This paper is structured in the following manner. In Section 2 we discuss the theoretical aspects of the framework. Section 3 is dedicated for literature survey and related work, and in Section 4 and 5 we define the ALMS framework and every module's functionality and implementation. Section 5 we conclude the paper.

\section{THEORETICAL BACKGROUND}

In this section, theoretical concepts about various aspects of the frame work have been elaborated.

\subsection{Automatic Learning Style Detection}

An adaptive system must be capable of managing learning paths adapted to each user, monitoring user activities, interpreting those using specific models, inferring user needs and preferences and exploiting user and domain knowledge to dynamically facilitate the learning process [1]. As per the definition by James and Blank [2] learning style is "the complex manner in which, and the conditions under which, learners most efficiently and most effectively perceive, process, store and recall what they are attempting to learn". The another definition of the same is learning styles represent a combination of cognitive, affective and other psychological characteristics that serve as relatively stable indicators of the way a learner perceives, interacts with and responds to the learning environment [3]. In this paper also the same notion has been adopted. There are two methods of finding leaner's style i.e., Finding learning style from the user input or automatic extraction of learner's style with less user input. In first method users fill length questionnaire and give their maximum input which makes the user to get into bored learning. This method is also called collaborative approach. Learners style may be detected automatically from the prior history of the learners, their behavior and interactions which is semi/fully automated method. The second method of detection of learning profile may be implemented using OWL - Web Ontology Language to represent the profile semantically and extract the same to dynamically set the workflow for a specific learner. The main goal of web 3.0 is personalization and this has become the vital feature in e-learning, Finding personalized workflow for a learner can be established using various ways. In our method we use Semantic Web technologies and Ontologies to develop the representation of learning objects, assessment Ontologies and competencies Ontologies that link learning with personal preferences and interest.

The necessities that led us to design ALMS

- facilitates the student to evaluate themselves

- Find themselves what kind of learner he/she is

- Tools and processes are required to obtain the correct workflow of LOs that lead to automatic dynamic workflow composition.

- Accessing LOs in knowledge based repository on the fly. 
- Designing cyclic LMS which takes feedback from learners to enhance the customized workflow

\subsection{Automatic composition of web services}

Downsizing the learning content to meaningful units with manual, semi-automated and automatic procedures is a tough process [4]. In web services architecture, WSDL-Web Service Description Language is used for web service description which takes syntactic aspects into consideration. Web services related operations are very difficult to achieve with these syntactic descriptions. But OWL-S profile and process ontologies are based on semantics using which integration, discovery and composition of web services are made easy. Manual composition of workflows, as any user driven process, is a task prone to errors and inconsistencies [5].

In e-learning processing, the learner who does not know about his/her requirements, and who's learning profile need to be found may not involve in composition of e-learning web services. By user profile, and their behavior the LMS has to find user requirements. Simply linking the input and output parameters in the web services will not help. Automation of this work flow composition helps him/her to relieve from the burden of deciding what services they need and it needs semantics to be described using OWL-S.

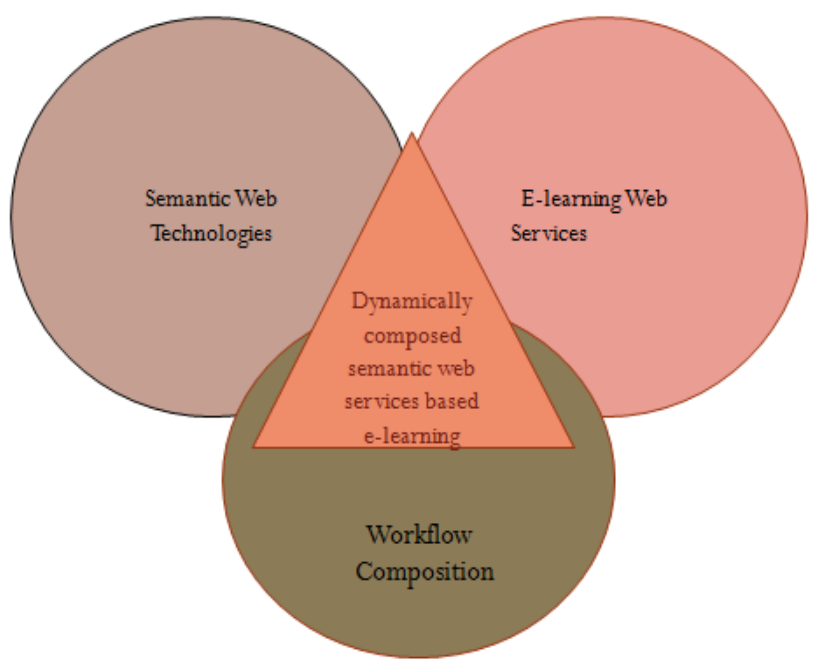

Figure 1. Conceptual Model

\subsection{Benefits of ALMS}

In our ALMS framework we combine the three aspects 1) Learning style detection 2) knowledge representation, and 3) Work flow composition which is conceptually represented in Figure 1 to provide the following benefits to any e-learner.

- Personalized workflow for dynamic learners whose behavior and requirements changes over time.

- Collaborating with people with similar experience

- Enforces continuous learning 
- Supports categories of learners styles

- Provides guidelines to Instruction designers to move toward with most appropriate design for their student community

\section{LITERATURE SURVEY AND RELATED WORK}

This section lists out the related work done by different approaches

\subsection{Learning Style Detection and Adaptive Learning Management System}

Proper understanding of Learning Style theory is required to design learning style based work flow composition in e-learning framework. Learning style models have been suggested by various researchers. They are VAK Model, Kolb's model, Honey and Mumford's model and FSM-Felder-Silverman model. In these models either collaborative or automatic approach has been adhered. Learning styles are based on the research outcomes of cognitive psychology about processing information, active learning and the structure of information [6]. The following is the list of work done in this automatic student modeling.

The first idea of providing personalized learning introduced formally by Koumi[19]. He implemented multimedia based adaptive learning management system in which computer offers individualized control and interactivity. Tailoring e-learning content to satisfy the individual is a challenging process. Nabila et al [8] suggested a method of learning style detection in which observable behaviors and actions of learners tracked and converted into style. Farman Ali Khan et al [9] tracked learners' knowledge progress, provides an assessment, and supports the learner by offering hints, help, and feedback in their proposed learning and assessment tool/framework. They discussed about affective state of learners plays major role in effectiveness of e-learning. [16] Dung and Florea concentrate on intelligent agents that can provide the learners with personal assistants to carry out learning activities according to their learning styles and knowledge level. Learner's interest, goals, and their prior knowledge in that subject are major concerns in any ALMS. In this kind of LMS, student centered approach is given more significance. The rationale behind adaptive LMS is that accommodating the individual differences of the learners (in terms of knowledge level, goals, learning style, cognitive abilities, etc.) is beneficial for the student, leading to an increased learning performance and/or learner satisfaction[1]. Online learners behavior cannot be predicted in advance and it changes over time. Wolf $C$ [10] has tested whether multimedia based Learning objects improved weak students performance or not. Melody Siadaty and Fattaneh Taghiyareh [11] used the Jackson's Learning Styles Profiler (LSP) [12] in order to model the learning styles of the learners. Jackson defined five learning styles: sensation seeker, goal oriented achiever, emotionally intelligent achiever, deep learning achiever and conscientious achiever [1]. In our ALMS, learning style module uses the technique of Sabine Graf model [17] and Jackson Learning Style Profiler and uses semantics and knowledge base of Ontologies to derive the learning styles in LSP [1]. We prefer to use this model because a learner will fit into any one of these categories

\subsection{Automatic workflow composition:}

Instruction design and instruction development are the two components of instruction technology. Learning objects development involves creation of different LOs by teaching and subject experts. LOs can be ppts, slides, multimedia based components, assessments and feedback module, 
simulation modules, etc. Instruction design involves how to offer these learning objects, and what is the basic criteria for providing the learning objects and e-learning services. The area of elearning consists of a multiplicity of complex activities, such as content authoring or learner tracking and administration which interact with resources (including people such as learners and authors), with one another (some activities trigger others), and with the outside world (such as existing software systems) in a predefined way [13]. Jihie Kim et al [5] explained about AI planning techniques in which rich knowledge base is used for automatic composition. They have come up with approach for interactive workflow composition that includes 1) knowledge-rich descriptions of the individual components and their constraints; 2) a formal algorithmic understanding of partial workflows, based on AI planning techniques. Eran Chinthaka et al [20] propose a Case-Based Reasoning (CBR) approach to assist composition of workflows based on the characteristics of the inputs and the outputs of the reusable workflow components, facilitating user exploitation of existing services and workflows during workflow composition.

Jorge Cardoso and Amit Sheth [14] has suggested QoS based workflow composition technique in which operational requirements timeliness, quality of products delivered, cost of service, and reliability are given significance for composition. Xubo Fei and Shiyong Lu [15] uses data flow driven approach for composing scientific workflow. In this paper they have suggested a dataflowbased scientific workflow model that separates the declaration of the workflow interface from the definition of its functional body; a set of workflow constructs, including Map, Reduce, Tree, Loop, and Conditional which are fully compositional one with another, a dataflow based exception handling approach to support hierarchical exception propagation and user-defined exception handling are the unique features of this paper.

\section{ALMS FRAMEWORK}

This framework in Figure 2. focuses on a cyclic model which includes continuous learning feedback-update-learning cycle. It seamlessly integrates the following modules:

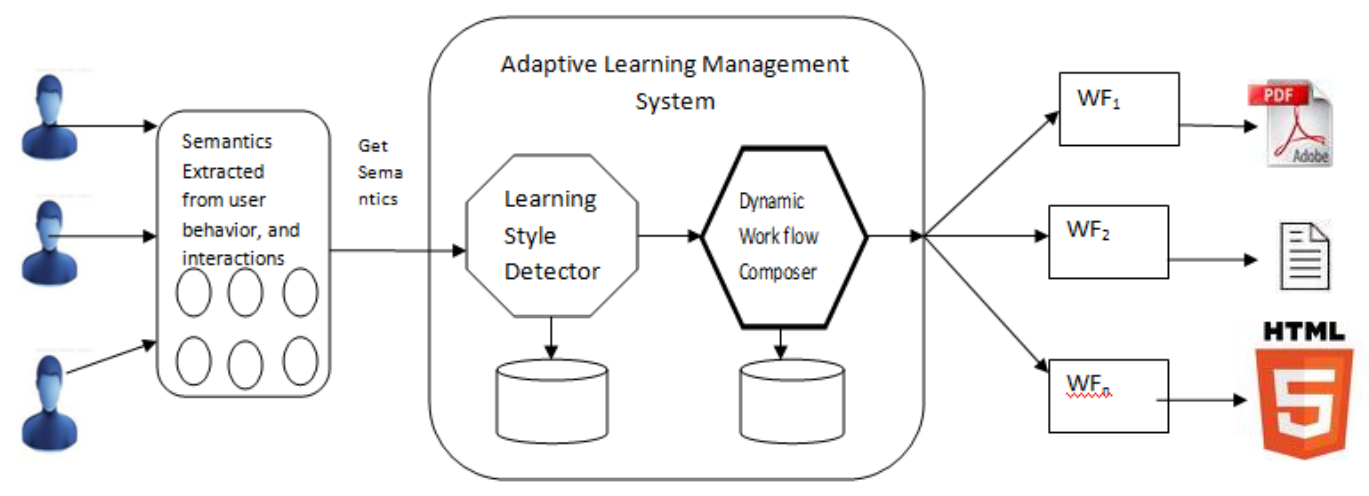

Figure 2. ALMS-Framework

ALMS framework can also be considered as QoS model which takes learners style as operational metric to decide the work flow of e-learning services. This framework has been divided into the following modules mentioned below. 
Input module: This module is based on the technique which uses mix of both collaborative and automatic concepts where the behavior, interactions and attitude of the learners are tracked based on certain list of activities. If the learner profile is available in knowledge base, that will also be obtained. Here the users are not directed to fill the lengthy questionnaire instead they perform certain activities (games kind) for that relevant subject learning. For example, user may be asked to list out certain keywords which are relevant to the subject.

Semantics Acquisition Module: From the user input Ontologies constructed and stored. Here the context ontology, learning object related ontologies such as i) Learner Ontology ii) Learner Style Ontology iii) Domain Ontology iv) Pedagogical Ontology v) Adaptation ontology vi) Workflow ontology are constructed. This module does not involve users. This module automatically detects available learning objects and takes the ontologies for that subject specified by the user.

Knowledge Extraction/Learners Style Detector Module: Ontologies obtained from knowledge acquisition module and profile/historical details of the learner are used to determine the kind of learner in other words learning preference of the learner is detected. [17] Sabine Graf approach is to be extended where learner's interaction and their behavior related ontologies stored in knowledge base and extracted to detect the Learning style. Ours uses the methodology where learner's behavior, actions, interactions and learner profile (if already exists in knowledge base) are stored in terms of Ontologies. The semantics obtained from the Ontologies are input for detecting learner's requirements. Ontology reasoners are involved to perform different operations such as union, intersection, merge and refinement on ontologies can be performed to find out the learning style of the e-learner [18].

Work flow composer module: Web services are independent entities. Achieving inter operability among heterogeneous web services is challenging task. The standards that are used for building independent, portable, inter operable web services are WSDL, SOAP and UDDI. But WSDL uses syntactical description of the web service. Workflow which is an abstraction of business process composed of one or more web services sequenced to resolve the business request. Syntactical nature of WSDL doesn't allow the composition or orchestration of these services statically or in other way with user intervention. Automatic composition can be achieved with Web Ontology language OWL-S in building semantic web services. Profile, grounding and process ontology are used to compose the services dynamically. The planner or composer in this is responsible for this workflow plan generation. There are several Heuristic approaches available. The one which we find suitable for dynamic composition is $A^{*}$ search algorithm. $A^{*}$ is which meant for best path finding is modified in our approach that takes an input of learning style detection and, identifies possible paths and returns the best workflow solution. This algorithm is similar to greedy best first algorithm which takes history of nodes traversed. Semantic web based E-learning services which provide different learning objects as a sequence will be identified for the learners preference. The composer which takes learning styles ontologies and learning Object ontologies use variant $\mathrm{A}^{*}$ algorithm to determine the best workflow.

Execution module: Realizes the work flow sequence obtained in composing module. Messaging happens to communicate among various entities in the sequence. Correct sequence of services executed and sequence will be provided to the Learners 


\section{IMPLEMENTATION}

This conceptual framework is under implementation in Jena Framework which is meant for creating semantic web application. The evaluation of this framework is to be conducted for various real time data. The results, findings and comparative study on various other ALMS will be carried out after the completion of the project.

\section{CONCLUSION}

Personalization is a vision of Web 3.0 which next generation web. Instruction design is an area which is still not given significance as far as semantic web concerned. Achieving this goal in elearning services is challenging one. In this paper we try to provide the theoretical aspects of combining two- three areas of our interest and bridge the gap between these fields. The conceptual framework ALMS combines learning style detection, ontology operations and knowledge management for reasoning and dynamic composition of e-learning services using planning algorithm to provide the benefits listed below

- suitable workflow for dynamic learners whose behavior changes over time.

- Collaborating with people with similar experience

- Enforces continuous learning

- Supports categories of learners styles

- helps the instruction designers to provide the most appropriate design for their student community

In this framework, we consider e-learning services into major consideration. But this approach can be applied to various business domains where dynamic composition is compulsory.

\section{REFERENCES}

[1] Popescu, E., Badica, C.,\& Moraret, L. "Accommodating Learning Styles in an Adaptive Educational System," Informatica, vol. 34 2010, no. Computational Linguistics and its Applications, p. 451-462, March 2010.

[2] Boticario, J G.,Santos, O C., Van Rosmalen P., "Issues in Developing Standard-based Adaptive Learning Management Systems," in EADTU 2005 Working Conference: Towards Lisbon 2010: Collaboration for Innovative in Lifelong Open and Flexible Learning, Italy, 2005.

[3] J. Keefe, "Learning Style: Overview," NASSP's Student Learning Styles: Diagnosing and Prescribing Programs, National Association of Secondary School Principles., pp. 1-17, 1979.

[4] Lytras, M., Sicilia, M., "Knowledge society a manifesto for knowledge and learning. Int. J. Knowledge and Learning 1(1), 1-11 (2005)

[5] J. Kim, M. Spraragen and a. Y. Gil, "An Intelligent Assistant for Interactive Workflow Composition," in Proceedings of the 9th international conference on Intelligent user interfaces IUI 04, Island of Madeira, Portugal, 2004.

[6] E. Kanninen, "Learning styles and e-learning," Tampere, 2008.

[7] Graf, S., Viola, S R., and Kinshuk, "Automatic Student Modelling for Detecting Learning Style Preferences in Learning Management Systems.," in In Proceedings of the Iadis International Conference on Cognition and Exploratory Learning in Digital Age, Algarve, Portugal, 2007.

[8] Bousbiaa, N., Jean-Marc L., Issam, R., Ballaa, A. "How to determine the Learners' Learning Styles in e-Learning Situation?," in Proceedings of The 16th International Conference on, 2008.

[9] F. A. Khan, E. R. Weippl and A. M. \& Tjoa, "Integrated Appraoch for the Detection of Learning Styles \& Affective States," in In G. Siemens \& C. Fulford (Eds.), Proceedings of World Conference 
on Educational Multimedia, Hypermedia and Telecommunications 2009, Honolulu, Hawaii, June 2009.

[10] Wolf, C., "Construction of an Adaptive E-learning Environment to Address Learning Styles and an Investigation of the Effect of Media Choice" RMIT University January 2007

[11] Siadaty, M., Taghiyareh, F. "PALS2: Pedagogically Adaptive Learning System based on Learning Styles," in Seventh IEEE International Conference on Advanced Learning Technologies (ICALT ), Niigata, Japan, 2008

[12] Jackson. C. [Online]. Available: http://www.cymeon.com.

[13] Westerkamp, P., "E-Learning as a Web Service," in 7th International Conference on Database Engineering and Applications (IDEAS), IEEE Computer Society Press, Hong Kong, China, 2003.

[14] J. Cardoso and Sheth. A., "Semantic e-Workflow Composition," Journal of Intelligent Information Systems (JIIS), vol. 2003. 21(3), pp. 191-225. , 2003.

[15] X. Fei and S. Lu., "A Data flow Based Scientific Workflow CompositioN framework," IEEE Transactions on Services Computing, vol. 5, no. 1, pp. 45-58, Jan-March 20122012.

[16] P. Q. Dung and A. M. Florea, "A literature-based method to automatically detect learning styles in learning management systems," in WIMS' 12 Proceedings of the 2nd International Conference on Web Intelligence, Mining and Semantics , Craiova, Romania, 2012.

[17] S. Graf, "Adaptivity in Learning Management Systems Focussing on Learning Styles," Austria, 2007. Ph.D thesis

[18] Farida Begam, M. and Ganapathy, G, "Knowledge Engineering Approach for constructing Ontology for e-Learning Services," in International Conference on Advanced Computer Science and Information System (ICACSIS 2011), IEEE explore Digital Library., Jakarta, Indonesia, 2011.

[19] Koumi, J. "Media comparison and deployment: a practitioner's view", British Journal of Educational Technology, 25(1), 41-57. 1994

[20] Chinthaka, Ekanayake, et al., "CBR Based Workflow Composition Assistant", Proceeding SERVICES '09 of the 2009 Congress on Services -I, , Pages 352-355, 2009.

\section{AUTHORS}

M. Farida Begam received her Bachelor of Engineering in Computer Science and Engineering from Bharathidasan University, India in 1998 and Master of Technology in National Institute of Technology, Trichy, India in 2006 and she worked as a Technical Evangelist in Infosys Technologies, India and now she is working as Senior Lecturer in the Department of Information Technology, Manipal University, Dubai Campus, U.A.E. She is currently pursuing Ph.D., in Bharathidasan University, India in the field of Semantic Web based E-learning. She is also CCNA Certified Instructor. Her research interests include Web technologies, Semantic Web, Computer Networks, Operating systems and Security.

Gopinath Ganapathy PhD is the Head, School of Computer Science Engineering and Applications, Bharathidasan University, India. He did his under graduation and postgraduation in Computer Science and Applications in 1986 and 1988 respectively from Bharathidasan University, India. He obtained his $\mathrm{PhD}$ degree, in Computer Science in 1996, from Madurai Kamaraj University, India. Received Young Scientist Fellow Award for the year 1994 and eventually did the research work at IIT Madras. He published around 20 papers. He is a member of IEEE, ACM, CSI, and ISTE. He was a Consultant for 8.5 years in the international firms in the USA and the UK, including IBM, Lucent Technologies (Bell Labs) and Toyota. His research interests include Semantic Web,

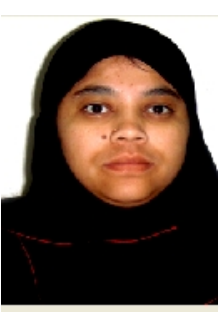
NLP, Ontology, and Text Mining.

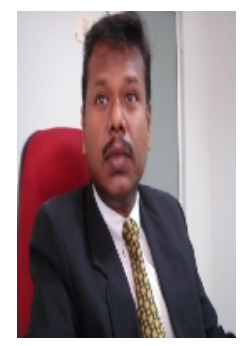

